\title{
Prevalência e variação dos estádios eritrocíticos do Plasmodium (Novyella) juxtanucleare em Gallus gallus sob condições naturais, no período de um ano
}

\author{
HELBA HELENA SANTOS-PREZOTO*, MARTA D`AGOSTO** e ERIK DAEMON** \\ PREVALENCE AND VARIATION OF THE ERYITROCYTIC FORMS OF Plasmodium \\ (Novyella) juxtanucleare IN Gallus gallus ABOUT NATURAL CONDITIONS, \\ IN THE PERIOD OF ONE YEAR
}

The avian malaria caused by Plasmodium juxtanucleare in Gallus gallus, is a tipical plasmodiose from Brazilian gallinaceous. This disease can causes morbidy and mortality in its vertebrate hosts. This work was conducted at Boa Vista farm, Municipality of Santa Bárbara do Tugúrio, Minas Gerais, Brazil and the ours objectives were to evaluate the hight prevalence found in previous studies and to accompany the variation of the erytrocytic forms during one year. The bloods smears, dyed with Giemsa were examined in microscopy immersion. Twenty five half-breed fowls were accompany duting one year, monthly (from November/00 to May/01) and biweekly (from June/01 to October/01). The erytrocytic forms were registered and quantified by the observation of 100 microscopic fields. Was verified a prevalence of $100 \%$ by $\boldsymbol{P}$. juxtanuclerare, but there wasn't statistics correlation between the increase of the erytrocytic forms during the year. The trophozoites were the more abundant form found in this studie.

Key words: prevalence, erytrocytic forms, Plasmodium juxtanucleare, fowls, natural conditions.

\section{INTRODUÇÃO}

Várias espécies de plasmódios causam a malária aviária, sendo as espécies Plasmodium (Novyella) juxtanucleare Versiani \& Gomes, 1941 e Plasmodium (Haemamoeba) gallinaceum Brumpt, 1935, os únicos causadores da malária em Gallus gallus ${ }^{1}$. Os mosquitos vetores incluem espécies das tribos Anophelini e Culicini para $P$. juxtanucleare 2,3 e mosquitos Culicini, para $P$. gallinaceum $^{2-7}$.
A malária aviária causada pelo $P$. juxtanucleare caracteriza-se por altas taxas de morbidade e mortalidade, podendo-se observar sintomatologia nervosa com paralisia em aves infectadas experimentalmente, causada por lesões do tipo necrótica ou inflamatória no cérebro e medula espinhal, ou por efeitos do parasito e seus produtos metabólicos no sistema nervoso central ${ }^{8}$.

Em infecções naturais, além da presença do quadro clínico, já foi relatado sinais de depressão, anemia, perda de apetite, fezes esverdeadas e baixa

\footnotetext{
* Curso de Pós-graduação em Ciências Biológicas - Comportamento e Ecologia Animal. Universidade Federal de Juiz de Fora. Campus Universitário, 36036-330, Juiz de Fora, Minas Gerais, Brasil. helba.santos@ig.com.br ** Departamento de Zoologia, Instituto de Ciências Biológicas, UFJF
} 
produção de ovos ${ }^{9}$, ocorrendo também em criações industriais ${ }^{10}$.

Alguns autores ${ }^{11-13}$ sugeriram que a malária aviária causada por $P$. juxtanucleare é uma doença que apresenta infecção crônica, assintomática e raramente fatal. Assim, os animais portadores deste hematozoário representam, na natureza, fontes de infecção para os vetores ${ }^{10}$. Todavia, autores ${ }^{14,15}$ mencionaram um quadro de anemia, febre, inapetência, megalia dos órgãos, distúrbios digestivos e neurológicos (sonolência, paralisia das patas, balançar de cabeça, tremores, prostação) e morte associada com o grau de parasitemia.

O parasitismo por $P$. juxtanucleare já foi relatado em vários países como Brasil ${ }^{4}$, México $^{16,17}$, Uruguaii ${ }^{18}$, Japão ${ }^{19,20}$, Sri Lanka ${ }^{21}$, Malásia $^{22}$, Ceilão ${ }^{23}$, Taiwan ${ }^{24}$, Tanzânia ${ }^{25}$ e Argentina ${ }^{26}$.

No Brasil, foram registrados a ocorrência de P. juxtanucleare nos Estados de Minas Gerais ${ }^{4,12,}$ ${ }^{27-30}$, Rio de Janeiro ${ }^{10,29,31-34}$, Espírito Santo ${ }^{31}$, Pará $^{35}$, Mato Grosso do $\mathrm{Sul}^{36}$ e Pernambuco ${ }^{37}$.

Visto que $\mathrm{Maia}^{30}$, em um estudo realizado no sítio Boa Vista, município de Santa Bárbara do Tugúrio/MG, detectaram prevalência de $100 \%$ de infecção de $P$. juxtanucleare em aves de criação rústica mantidos em condições naturais, este fato motivou a realização de novas análises no local. Assim o presente trabalho teve como objetivos reavaliar a prevalência de $100 \%$ encontrada em estudos anteriores; acompanhar a variação dos estádios eritrocíticos do parasito ao longo de um ano e verificar a média de campos positivos para $P$. juxtanucleare na região.

\section{MATERIAL E MÉTODOS}

Foram acompanhadas 25 aves mestiças de criação rústica, mantidas em área rural do Sítio Boa Vista, município de Santa Bárbara do Tugúrio

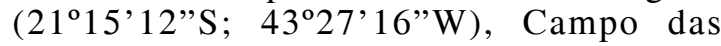
Vertentes, Estado de Minas Gerais. As aves foram mantidas em áreas abertas e alimentadas com milho, frutas e pastagem, permanecendo soltas durante todo o dia. O local não recebeu nenhum tipo de cuidado referente à higiene e nem medidas profiláticas contra doenças aviárias. É importante informar que os exemplares não apresentaram sinais clínicos de qualquer tipo de doenças infecto-contagiosa ou parasitária.

Os dados foram coletados no período de novembro/2000 a outubro/2001, totalizando 17 coletas realizadas, sendo sete mensais (de novembro a maio) e dez quinzenais (de junho a outubro).

Os indivíduos foram marcados por "pulseiras de plásticos" numeradas denominadas "lacre para malotes", que foram fixadas em uma das pernas, determinando para cada ave uma numeração individual.

Os dados foram incluídos em uma ficha de identificação contendo a data de coleta, temperatura do ambiente, umidade relativa do ar, aspectos físicos e comportamentais dos hospedeiros, idade, sexo, peso e temperatura corporal.

As amostras sangüíneas foram obtidas por venopunção radial, utilizando-se agulhas descartáveis, e confeccionados esfregaços finos de sangue periféricos, fixados em metanol por três minutos. Cada lâmina recebeu a numeração referente ao código da ave amostrada.

No Laboratório de Microscopia do Curso de Pós-Graduação em Ciências Biológicas (Zoologia)Comportamento e Ecologia Animal, da Universidade Federal de Juiz de Fora, os esfregaços foram corados pelo Giemsa (Eosina Azul de Metileno) diluído em tampão de Sorensen pH $6,8^{38} \mathrm{e}$, posteriormente analisados. Os estádios eritrocíticos foram quantificados diferencialmente em trofozoítos, esquizontes, microgametócitos e macrogametócitos, pela observação de 100 campos homogêneos para 10.000 hemácias $^{42}$ em imersão, em microscopia fotônica (OLYMPUS BX50).

A identificação e caracterização morfológica de $P$. juxtanucleare foi realizada segundo descrição de vários autores ${ }^{1,2,6,39}$.

\section{RESULTADOS E DISCUSSÃO}

\section{1.- Prevalência de Plasmodium juxtanucleare ao} longo de um ano: A prevalência de $P$. juxtanucleare no período estudado foi de $100 \%$, valor muito elevado no que se refere a uma plasmodiose. Em estudo anterior ${ }^{30}$, no mesmo local, já haviam verificado esta prevalência de $100 \%$; tal fato indica que nos últimos anos vem ocorrendo uma constante e elevada manutenção desse parasitismo no local.

Somente um trabalho ${ }^{33}$ obteve também $100 \%$ de prevalência em quatro criações do Estado do Rio de Janeiro, sendo uma na Mesorregião do 


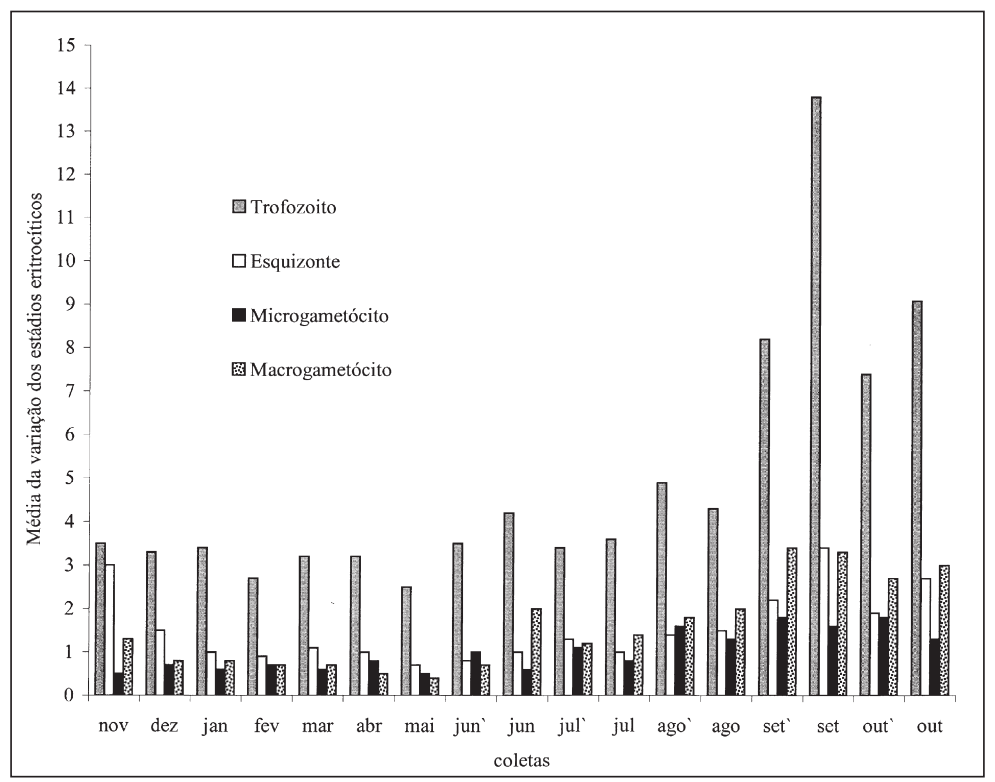

Figura 1. Média dos estádios eritrocíticos, em 100 campos examinados, de Plasmodium juxtanucleare em 17 coletas realizadas de novembro/00 a outubro/01, no sítio Boa Vista, município de Santa Bárbara do Tugúrio, Minas Gerais, Brasil.

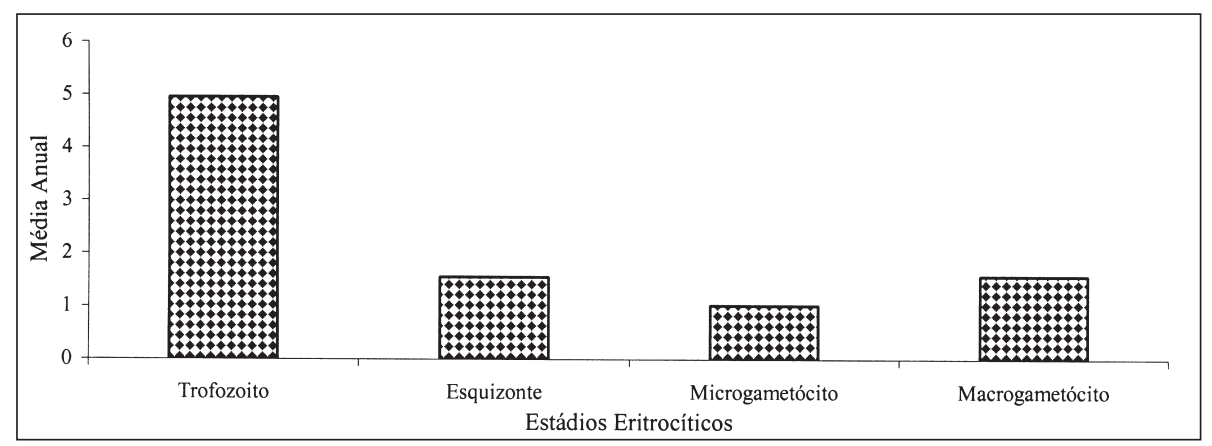

Figura 2. Média anual dos estádios eritrocíticos de Plasmodium juxtanucleare em 25 aves estudadas de novembro/00 a outubro/01, no Sítio Boa Vista, município de Santa Bárbara do Tugúrio, Minas Gerais, Brasil.

Vale do Paraíba, duas no Norte Fluminense e uma na periferia do Grande Rio. Em criações industriais foi diagnosticado ${ }^{10}$ prevalência de 79,5\% em galinhas da raça Leghorn Branca, devido à elevada concentração de hospedeiros vertebrados na área. Já alguns autores ${ }^{35}$ detectaram em Belém (PA) prevalência de $40 \%$, enquanto outros ${ }^{1}$ obtiveram índices de $39,7 \%$ no Estado do Rio de Janeiro e 39,2\% no Estado do Espírito Santo. Mota ${ }^{13}$ obtiveram índice de 28,4\% para 250 aves analisadas da Região Metropolitana do Recife, PE. Paraense ${ }^{28}$ obteve uma prevalência de 21,3\% na região de Bambuí, MG, enquanto Krettli ${ }^{6}$ em várias regiões do Estado de Minas Gerais obteve $18 \%$ para Bambuí, 33\% para Belo Horizonte, $8 \%$ Betim, 4\% Igarapé e $9 \%$ para Peçanha, Sabinópolis e Governador Valadares. Pesquisadores ${ }^{29}$ verificaram prevalência de 2,34\% em aves procedentes do Rio de Janeiro e 1,38\% de Minas Gerais. Já outros ${ }^{13}$ obtiveram prevalência de 1,4\% em aves do Estado do Pernambuco.

Pode-se perceber que existe uma ampla variação entre as prevalências encontradas nas 


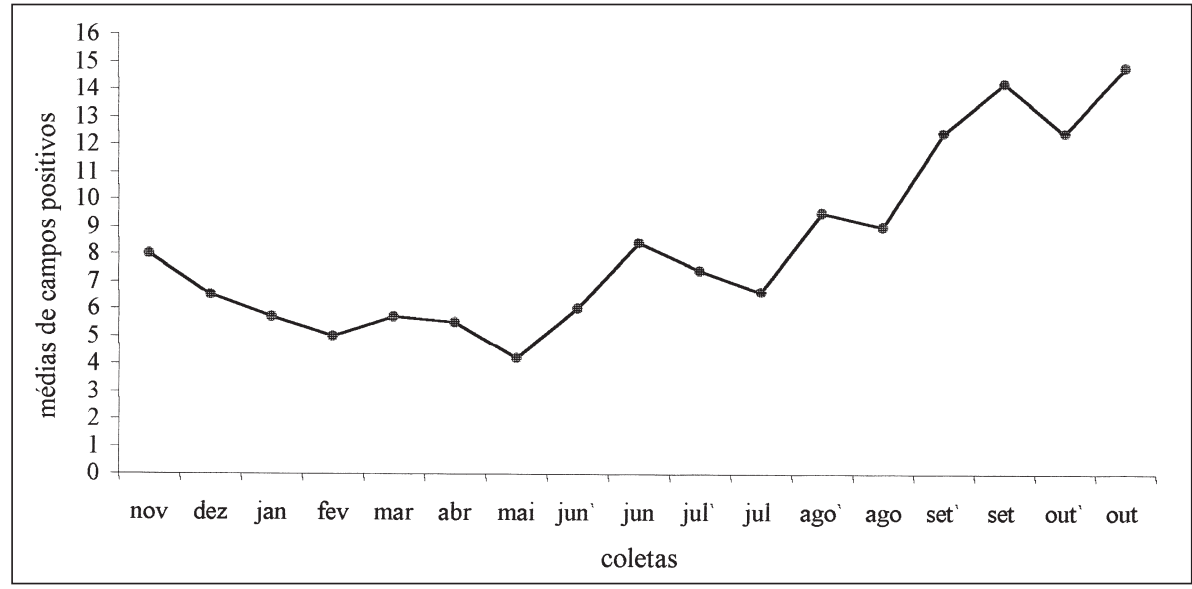

Figura 3. Média de campos positivos em 100 campos examinados, para Plasmodium juxtanucleare em 25 aves, em 17 coletas de novembro/00 a outubro/01, no sítio Boa Vista, município de Santa Bárbara do Tugúrio, Minas Gerais, Brasil.

mais variadas regiões brasileiras. No entanto, na região do Campo das Vertentes (MG) foi detectada, desde 1999, elevada prevalência de $100 \%$, que pode estar relacionada à quantidade $\mathrm{e}$ a sazonalidade do mosquito vetor do $P$. juxtanucleare no local estudado, bem como da presença de gametócitos no sangue das aves durante todo o ano.

\section{2.- Variação dos Estádios Eritrocíticos do $P$. juxtanucleare:}

2.1. Médias dos Estádios Eritrocíticos por Coleta

No presente estudo pôde-se registrar a ocorrência dos quatros estádios eritrocíticos de $P$. juxtanucleare (trofozoítos, esquizontes, microgametócitos e macrogametócitos) em todas as coletas do período estudado, já em aves com parasitemia mais elevada pôde-se verificar a ocorrência de poliparasitismo.

De uma maneira geral, os trofozoítos foram os mais encontrados, fato que fica mais evidentes principalmente nas quatro últimas coletas (set 8,2; set 13,8; out' 7,4 ; out 9,1) (Figura 1).

Estatisticamente não houve significância da variação no número de trofozoítos em relação ao período de estudo, apesar de ter-se encontrado um indivíduo com 168 trofozoítos na coleta do mês de setembro (set). No entanto, o teste indicou variação entre as coletas de fevereiro e setembro (set), fevereiro e outubro (out), maio e outubro (out), abril e outubro (out), maio e setembro (set) e maio e outubro (out).
As médias das coletas de novembro $(3,0)$, setembro (2,3 e 3,4 respectivamente) e outubro (out 2,7) caracterizaram-se pelo elevado índice de esquizontes em relação aos demais meses. $\mathrm{O}$ menor valor obtido para esquizontes foi de 0,7 em maio, e o maior de 3,4 em setembro (set). Estatisticamente não houve uma tendência de aumento do número de esquizontes durante as 17 coletas, no entanto os dados indicam uma variação das coletas dos meses de fevereiro e outubro (out), março e outubro (out), abril e outubro (out), maio e agosto (ago'), maio e setembro (set) e entre maio e outubro.

O estádio gametócito foi o menos encontrado se comparado com trofozoítos e esquizontes. Macrogametócito apresentou sua maior média em setembro (set`3,4) e microgametócito apresentou índice mais elevado em outubro (out' 1,8 ), no entanto um trabalho ${ }^{40}$ obteve números de microgametócitos superior ao de macrogametócitos de $P$. juxtanucleare, em condições experimentais.

Tanto para macrogametócito quanto para microgametócito o teste de Friedman não indicou aumento do número dos estádios durante o período experimental, sugerindo que em qualquer período do ano pode-se encontrar gametócitos no sangue das aves estudadas. Este fato pode explicar porque mesmo com uma baixa parasitemia houve uma elevada prevalência no local. Esta observação concorda com Krettli ${ }^{12}$ que, apesar de ter encontrado baixa parasitemia em infecções naturais, observou que os gametócitos foram facilmente encontrados, explicando a ampla 
distribuição do parasito e assegurando a transmissão.

\subsection{Média Anual dos estádios eritrocíticos $P$.} juxtanucleare

A média de trofozoítos encontrados durante todo o período de coleta foi de 4,9 , sendo um valor muito alto em relação aos demais estádios, que foram os seguintes: 1,5 para esquizontes, 1,6 para macrogametócitos e de 1,0 para microgametócitos (Figura 2).

O maior número de trofozoítos em relação aos demais estádios eritrocíticos, encontrado no presente trabalho concorda com os dados obtidos por outros trabalhos ${ }^{12}$, em infecções naturais, com 134 trofozoítos, 71 esquizontes e 15 gametócitos, não especificando se microgametócito ou macrogametócito. Oliveira ${ }^{40}$, em condições experimentais, após 40 dias de inoculação, obteve o total de 106 trofozoítos, 98 esquizontes, 18 macrogametócitos e 34 microgametócitos.

\subsection{Média de Campos Positivos Para $P$. juxtanucleare}

A média de campos positivos para 100 campos analisados por coleta, em relação aos estádios eritrocíticos de $P$. juxtanucleare em galinhas domésticas, variou durante todo o período de estudo. No entanto, o maior índice encontrado foi de 14,8 em outubro (out) e menor e de 4,2 em maio (Figura 3).

O período no qual se registrou o maior número de formas eritrocíticas nas coletas pode estar relacionado com o pico reprodutivo dos mosquitos, o que se deve à proximidade do verão, com elevação da temperatura e aumento da pluviosidade no ambiente, proporcionando condições perfeitas para a reprodução do mosquito vetor. Alguns autores ${ }^{41}$ citam que a temperatura ambiente ótima para o desenvolvimento das larvas da maioria das espécies de mosquitos varia de 24 a $28^{\circ} \mathrm{C}$, concordando com as médias de temperatura obtidas no presente trabalho $\left(26,6^{\circ} \mathrm{C}\right.$ em setembro e $24,6^{\circ} \mathrm{C}$ em outubro).

É importante ressaltar que apesar de terem sido detectados valores elevados deste plasmódio no sangue das aves, nenhuma apresentou sintomas característicos da malária aviária, o que pode indicar uma virulência baixa da cepa e/ou uma resistência imunológica das aves quanto à referida cepa de $P$. juxtanucleare encontrada no local.

Estatisticamente, não houve aumento significativo do número de estádios eritrocíticos durante o ano de estudo, revelando haver significância apenas entre as coletas de fevereiro e outubro (out), abril e maio, abril e outubro (out) e maio e outubro (out), o que indica um possível aumento de campos positivos e, conseqüentemente aumento do número de parasitos no sangue entre as primeiras coletas e as últimas. Faz-se necessário a realização de um maior período de acompanhamento no local para maior análise sobre o significado do aumento da parasitemia nesse período do ano.

\section{RESUMO}

Este trabalho sobre a malária aviária causada pelo Plasmodium juxtanucleare em Gallus gallus no Brasil, foi realizado no sítio Boa Vista (21 ${ }^{\circ} 15^{\prime} 12^{\prime}$ 'S , 4327'16”W), município de Santa Bárbara do Tugúrio, Minas Gerais, e teve como objetivos reavaliar a prevalência de $100 \%$ encontrada em estudos anteriores e acompanhar a variação dos estádios eritrocíticos do parasito ao longo de um ano na região. Sob microscopia em imersão foram examinados esfregaços sangüíneos de 25 aves mestiças, corados pelo Giemsa. As amostras foram coletadas ao longo de um ano, mensalmente de novembro/00 a maio/ 01 e quinzenalmente de junho/01 a outubro/01. Os estádios eritrocíticos foram quantificados pela observação de 100 campos microscópicos. Foi constatada prevalência de $100 \%$ por $P$. juxtanuclerare na região. Não houve correlação estatisticamente significativa entre o aumento dos estádios eritrocíticos em relação às coletas ao longo do ano. O estádio de trofozoíto foi predominante durante todo o período de estudo.

\section{REFERÊNCIAS}

1.- MASSARD C L, MASSARD C A. Aspectos biológicos do Plasmodium (Novyella) juxtanucleare (Versiani \& Gomes, 1941 em aves do Brasil. Rev Bras Med Vet 1981; 4: 3-24.

2.- GARNHAM P C C. Malaria Parasites and Other Haemosporidia. Blackwell Sci. Public. Oxford, 1114 p. 1966.

3.- KELLY R, EDMAN J. E. Multiple transmission of Plasmodium gallinaceum (Eucoccida: Plasmodiidae) during serial probing by Aedes aegypti (Diptera: Culicidae) on several hosts. J Méd Entomol 1992; 29 : 329-31. 
Plasmodium (Novyella) juxtanucleare em Gallus gallus - H. H. Santos-Prezoto et al.

4.- VERSIANI V, GOMES B F. Sobre um novo hematozoário de galinha, Plasmodium juxtanucleare n.sp. (nota prévia). Rev Bras Biol 1941; 1: 231-3.

5.- BENNETT G F, EYLES D E, WARREN M C W, CHEONG W H. Biology of the malaysian strain of Plasmodium juxtanucleare Versiani \& Gomes, 1941. II- The sporogonic stages in Culex (Culex) sitiens Wiedmann. J Parasitol 1966; 52: 647-52.

6.- KRETTLI A U. Plasmodium juxtanucleare in the State of Minas Gerais, Brazil. Studies on its prevalence and some aspects of its biology. Rev Inst Med Trop São Paulo 1972; 14: 235-45.

7.- LOURENÇO-DE-OLIVEIRA R, CASTRO FA. Culex saltanensis Dyar, 1928 - Natural vetor of Plasmodium juxtanucleare in Rio de Janeiro, Brasil. Mem Inst Oswaldo Cruz 1991; 86: 87-94.

8.- AL DABAGH M A. Symptomatic partial paralysis in chicks with Plasmodium juxtanucleare. J Comp. Pathol 1961; 71: 217-21.

9.- ITAGAKI K. An avian malaria in Japan. J Parasitol 1970; 56: 164.

10.- MASSARD C L. Caracterização do Parasitismo por Plasmodium juxtanucleare (Haemosporidea: Plasmodiidae) em Criações de Gallus gallus da Raça Leghorn Branca. Arq Univ Fed Rur Rio de Janeiro Itaguaí 1982; 5: 141-6.

11.- PARAENSE W L. Observações preliminares sobre o ciclo exoeritrocitário do Plasmodium juxtanucleare Versiani e Gomes, 1941. Mem Inst Oswaldo Cruz 1947; 45: 813-23.

12.- KRETTLI A U. Estudos sobre prevalência, biologia e transmissão do Plasmodium juxtanucleare Versiani \& Gomes, 1941. Tese de Mestrado, Universidade Federal de Minas Gerais, Belo Horizonte, 73p. 1971.

13.- MOTA R A, CUNHA E L P, SOARES C O et al. Variáveis Hematológicas em Gallus gallus domesticus, Linneaus, 1758, de criações rústicas da região metropolitana do Recife, naturalmente infectados com Plasmodium juxtanucleare (Versiani \& Gomes, 1941). Ciênc Vet Trop Recife 1998; 1: 76-80.

14.- SOARES C O, FONSECA A H, MASSARD C L, SOUZA P C A. Significância da Malária Aviária em Gallus gallus L. de criações rústicas. Anais do Seminário Brasileiro de Parasitologia Veterinária, Campo Grande, Mato Grosso do Sul. 201p. 1995.

15.- SOARES CO, MASSARD C L, FONSECA A H, SOUZA P C A. Esquizogonia exoeritrocitária plasmática em Plasmodium (Novyella) juxtanucleare (Apicomplexa: Plasmodiidae). Parasitol al Día 1999; 23: 87-90.

16. -BELTRAN E. Hallazgo de Plasmodium juxtanucleare Versiani \& Furtado em gallinas de Chiapas. Rev Inst Salub Enferm Trop Mex 1941; 2: 353-4.

17.- BELTRÁN E. Característica de uma cepa mexicana (14B) de Plasmodium juxtanucleare Versiani \& Gomes, 1941. Rev Inst Salub Enferm Trop Mex 1943; 4: 265-72.

18.- CASSAMAGNAGHI A. Malária em las aves del Uruguay. Montevideo, Fac. Vet., 93p. 1947.

19.- ISHIGURO H. Plasmodium japonicum, a new specie of malaria parasite pathogenic for the domestic fowl. Bull Fac Agri Yamaguti Univ 1957; 8: 723-32.

20.- AKIBA K. Studies on avian malaria. On the species of Plasmodium from chicken in Japan $(P$. japonicum
Ishiguro, 1957 a synonym for $P$. juxtanucleare Versiani \& Gomes, 1941). Jap J Vet Sci 1959; 21: 18.

21.- DHANAPALA S B. The ocurrence of Plasmodium juxtanucleare Versiani \& Gomes, 1941 in domestic fowls in Ceylon. Rev Malar 1962; 41: 39-46.

22.- BENNETT G F, EYLES D E, WARREN M C W, CHEONG W H. Plasmodium juxtanucleare a newly discovered parasite of domestic fowl in Malasya. Singapore Med J 1963; 4: 172-3.

23.- DINASSANAIKE A S. On some blood parasites of wild animals in Ceylon. Ceylon Vet 1963; 14: 29-37.

24.- MANWELL R A. Plasmodium japonicum, Plasmodium juxtanucleare and Plasmodium nucleophilum in the far east. J Protozool 1966; 13: 811.

25.- MOHAN RN, MANWELL RD. Plasmodium juxtanucleare in African partridges (Francolinus sp.). J Parasitol 1966; 55: 543.

26.- BOERO J J. Parasitoses animales. Tomo II, Ediciones Previas, Eudeba, Argentina, 89-264p.

27.- VERSIANI V, GOMES B F. 1943. Plasmodium juxtanucleare parasita de galinha doméstica (notas adicionais). Rev Bras Biol 1974; 3: 113-7.

28.- PARAENSE W L. Um inquérito sobre a ocorrência de Plasmodium juxtanucleare em Bambui (estado de Minas Gerais). Mem Inst Oswaldo Cruz 1949; 47: 361-5.

29.- FERRAZ-FRANCO H, VAITSMÁN J, MOUSSATCHÈ I. Hemoparasitos em aves domésticas. Observações em matadouro do Distrito Federal. Rev Mil Rem Vet 1954; 14: 29-37.

30.- MAIA M C, SIQUEIRA I C V, SANTOS H H, d’Agosto M. Ocorrência de Plasmodium juxtanucleare Versiani \& Gomes, 1941 em Gallus gallus L. de criação rústica no município de Santa Bárbara do Tugúrio-MG. Anais do XI Seminário Brasileiro de Parasitologia Veterinária. 213p. 1999.

31.- MASSARD C L. Aspectos biológicos de Plasmodium juxtanucleare Versiani \& Gomes, 1941 em aves do Brasil. Tese de Mestrado. Universidade Federal Rural do Rio de Janeiro, Itaguaí, Rio de Janeiro, 53p. 1976.

32.- MASSARD C L. Significância das infecções causadas por Plasmodium (Noyella) juxtanucleare Versiani \& Gomes, 1941 (Haemosporida: Plasmodiidae) em Gallus gallus L. de criação industrial no estado do Rio de Janeiro. Tese de Doutorado. Universidade Federal Rural, Rio de Janeiro, 57p. 1979.

33.- SOUZA P C A. Malária Aviária: Parasitismo por Plasmodium (Novyella) juxtanucleare Versiani \& Gomes,1941 em Gallus gallus L. de criações rústicas, nas mesorregiões do estado do Rio de Janeiro e aspectos clínicos e patológicos de sua infecção experimental. Tese de Doutorado, Universidade Rural do Rio de Janeiro, Seropédica, Rio de Janeiro,137p. 1998.

34.- SOARES C O, SOUZA P C A, MOTA R A et al. Parasitismo de leucócitos e trombócitos de Gallus gallus L. por Plasmodium (Novyella) juxtanucleare (Apicomplexa: Plasmodiidae). Parasitol al Día 1999; 23: 44-7.

35.- SERRA FREIRE N M, MASSARD C L. Ocorrência e incidência de Plasmodium juxtanucleare Versiani \& Gomes, 1941, em Belém - Pará. Congresso Sociedade Brasileira Parasitol Anais Belém, SBP. 1976.

36.- SERRA FREIRE N M, MASSARD C L. Plasmodium 
juxtanucleare Versiani \& Gomes, 1941, parasita de Gallus gallus L., Meleagris gallopavo L. e Crysolophus spp. na região do Pantanal do estado do Mato Grosso do Sul. Atas. Sociedade de Biologia do Rio de Janeiro 1979; 20: 45-8.

37.- MOTA R A. Variações hematológicas em Gallus gallus domesticus Linnaeus, 1758, de criações rústicas da região metropolitana do Recife, naturalmente infectados com o Plasmodium (Novyella) juxtanucleare Versiani \& Gomes, 1941. Tese de Doutorado, Universidade Federal Rural do Rio de Janeiro, Seropédica, Rio de Janeiro, 58p. 1997.

38.- LUBINSKY G. Note on the use of Mcllvaine's buffer solutions in staining blood protozoa. Can J Zool 1960; 38: 437-8.

39.- BENNETT G F, WARREN M C W. Biology of the malaysian strain of Plasmodium juxtanucleare Versiani
\& Gomes, 1941. III- Life cycle of the erythocytic parasite in the avian host. J Parasitol 1966; 52: 653 9.

40.- OLIVEIRA C E. Criopreservação e caracterizações morfológica, morfométrica e ultra-estrutural de Plasmodium (Novyella) juxtanucleare Versiani \& Gomes, 1941 (Apicomplexa: Plasmodiidae). Dissertação de Mestrado, Universidade Federal Rural do Rio de Janeiro, Seropédica, Rio de Janeiro, 64p. 2001.

41.- CONSOLI R A G B, LOURENÇO-DE-OLIVEIRA R. Principais mosquitos de importância sanitária no Brasil. Editora Fiocruz. 1 ${ }^{\text {a }}$ Reimpressão. 225p. 1998.

42.- SOUZA P C A, SOARES C O, FREIXO A P A et al. Proposta para padronização do cálculo da parasitemia em infecções pelo Plasmodium juxtanucleare. Anais do XI Seminário Brasileiro de Parasitologia Veterinária. 213p. 1999.

\section{ATENCION A LOS AUTORES}

PARA AGILIZAR LA EDICION DE PARASITOLOGIA LATINOAMERICANA, LOS AUTORES DEBERAN ENVIAR POR CORREO LOS ARTICULOS CIENTIFICOS GRABADOS EN UN DISKETTE DE $31 / 2$ EN WORD 6.0 O SUPERIOR JUNTO CON EL MANUSCRITO DEL TRABAJO A PUBLICAR O ADJUNTARLO POR CORREO ELECTRONICO A halcaino@uchile.cl 